\title{
Effect of Granulated Liming Material on Soil Properties and Yield of Paddy in Acid Soil of Bramhavara
}

\author{
Ganapathi $^{{ }^{*}}$, K.S. Niranjana ${ }^{2}$, S.M. Jayaprakash ${ }^{3}$ and S. Harshitha ${ }^{1}$
}

${ }^{1}$ Department of Soil Science and Agricultural Chemistry, College of Agriculture, Navile, Shivamogga, ${ }^{2}$ AHRS, Thirthahalli, ${ }^{3}$ ZAHRS, Brahmavara, University of Agricultural and Horticultural Sciences, Navile, Shivamogga, India

*Corresponding author:

Keywords

Granulated liming material paddy, Acid soil, Bramhavara

Article Info

Accepted:

15 March 2019

Available Online:

10 April 2019
A field experiment was conducted during kharif 2017-18 at ZAHRS, Bramhavara to study the effect of granulated liming material on soil properties and yield of paddy. Treatments included lime application as granulated lime and agricultural lime at different combinations along with FYM and RDF were studied in this experiment. The results revealed that the treatment receiving $\mathrm{RDF}\left(60: 30: 60 \mathrm{Kg} \mathrm{N}, \mathrm{P}_{2} \mathrm{O}_{5}, \mathrm{~K}_{2} \mathrm{O} / \mathrm{ha}\right)+\mathrm{FYM}(10$ $\mathrm{t} / \mathrm{ha})+50$ percent lime requirement through granulated lime based on 45 per-cent $\mathrm{Ca}$ saturation (1.14 tonnes/ha) recorded significantly higher growth parameters, yield, $\mathrm{B}: \mathrm{C}$ ratio, soil $\mathrm{pH}$, available $\mathrm{N}, \mathrm{P}_{2} \mathrm{O}_{5}, \mathrm{~K}_{2} \mathrm{O}$ and $\mathrm{S}$.

\section{Introduction}

Liming materials are commonly applied to reduce the acidity and to increase productivity of soils. Correction of soil $\mathrm{pH}$ is the cornerstone of a successful crop nutrient management program. Soil $\mathrm{pH}$ affects nutrient solubility and influences the sorption or precipitation of nutrients like $\mathrm{Al}, \mathrm{Mn}$, and $\mathrm{Fe}$. Liming increase the $\mathrm{pH}$ of acidic soils and improves macronutrients availability of soil while reducing the solubility of $\mathrm{Al}$ and $\mathrm{Mn}$. Pelletized lime is finely ground limestone, which is made into small pellets for broadcasting with fertilizer. In pelletized form the lime is protected from wind drift, and the uniform texture eliminates any larger, nonreactive particles that can take years to fully break down within the soil (Alvarez et al., 2009). Clay or synthetic binders, such as lignosulfonates hold the pellets together and dissolve in contact with rainfall or soil solution, breaking down by solubilization or microbial action. The pellets are durable enough to withstand transport, and minimal dust is created on spreading. Finer liming 
materials dissolve and react more rapidly. Scott et al., (1992) investigated the most effective particle size (between 0.005 and 3 $\mathrm{mm}$ ) of liming material in an acid soil in Australia and concluded that the finest limestone particles produced greatest increases of $\mathrm{pH}$, exchangeable $\mathrm{Ca}$ and wheat (Triticum aestivum L.) production. For crop production, however, soil acidity is a complex of numerous factors involving nutrient/element deficiencies and toxicities, low activity of beneficial microorganisms and reduced plant root growth that limit nutrient and water uptake (Fageria and Baligar, 2003). The situation is further complicated by various interactions among these factors (Foy, 1992). The practice of correcting soil acidity reduces the available contents of $\mathrm{Al}, \mathrm{Fe}, \mathrm{Mn}$, $\mathrm{Zn}$, and $\mathrm{Cu}$, but increases the availability of other essential nutrients. Liming is an effective and dominant practice to raise soil $\mathrm{pH}$ and reduce acidity-related constraints to improve crop yields (Fageria \& Baligar, 2008). The objectives of the present study was to determine the finely grounded granulated limestone on chemical properties in soil and yield of paddy.

\section{Materials and Methods}

The field experiment conducted during kharif 2017-18 at ZAHRS, Bramhavara. The paddy variety $\mathrm{MO}_{4}$ was selected as test variety. The soil of the experimental site was having $\mathrm{pH}$ 4.86. The available $\mathrm{N}\left(191 \mathrm{~kg} \mathrm{ha}^{-1}\right)$ and $\mathrm{P}_{2} \mathrm{O}_{5}$ $\left(19.9 \mathrm{~kg} \mathrm{ha}^{-1}\right)$ content was low while that of $\mathrm{K}_{2} \mathrm{O}\left(225.0 \mathrm{~kg} \mathrm{ha}^{-1}\right)$ was found medium. The exchangeable $\mathrm{Ca}$ and $\mathrm{Mg}$ content were 2.90 and $1.40 \mathrm{cmol}(\mathrm{p}+) \mathrm{kg}^{-1}$, respectively and the available $\mathrm{S}$ was $15 \mathrm{mg} \mathrm{kg}^{-1}$ (Table 1).

The testing trial was carried out in RCBD design with 13 treatments and replicated three times. Gross plot size of the experiment was $4 \mathrm{~m}^{2}$. Liming materials were applied 15 days before transplanting as per the treatments.
Soil samples were collected before and after treatment imposition and analyzed for soil chemical properties as per standard procedure. Growth and yield attributes were recorded. Soil $\mathrm{pH}$ was determined 1:2.5 soil to water suspension by potentiometric method (Jackson, 1973). Available nitrogen in the soil was determined by alkaline potassium permanganate method as described by Subbiah and Asija (1956). Available phosphorus were extracted using Olsen's extractant for neutal and alkaline soils and Bray's extractant for acid soils and was determined by spectrophotometer (Jackson, 1973). Available potassium extracted using neutral normal ammonium acetate was determined by using flame photometer (Jackson, 1973). The exchangeable calcium and magnesium were determined by Versenate titration method (Jackson, 1973). Available sulphur was extracted from soil using 0.15 per cent calcium chloride solution and determined turbidimetrically as described by Black (1965). Fertilizer and manure applied as per package of practices (POP).RDF: 60:30:60 kg NPK/ha and FYM: 10t/ha.

\section{Results and Discussion}

\section{Effect of liming materials on soil chemical properties and available nutrient status}

\section{pH}

The treatment which received granulated lime recorded significantly higher soil $\mathrm{pH}$ in all the treatments studied as compared to powdered agriculture lime applied plots (Table 2). Granules of liming materials hold longer duration in the soil to react fully. Granulated lime holds the soil $\mathrm{pH}$ values higher for longer duration due to higher neutralization power and reduction of loss in leaching. As limestone is a source of $\mathrm{Ca}$ and $\mathrm{Mg}$ and in the presence of water the carbonates dissolve and 
the $\mathrm{OH}^{-}$and $\mathrm{HCO}_{3}{ }^{-}$ions are released, reducing soil acidity (Havlin et al., 1999).

\section{Available Nitrogen, $\mathrm{P}_{2} \mathrm{O}_{5}$ and $\mathrm{K}_{2} \mathrm{O}$}

Granulated lime applied plots recorded significantly higher available $\mathrm{N}, \mathrm{P}_{2} \mathrm{O}_{5}, \mathrm{~K}_{2} \mathrm{O}$ compared to plots receiving powdered agriculture lime in all the growth stages of crop. This was due to higher neutralization, less loss in soil, more reactivity and quality of granulated lime. Increase in soil $\mathrm{pH}$ from acidic condition by application of liming material leads to increase in availability of nutrients. Liming increases the beneficial microbial activity of the soil with increase in $\mathrm{pH}$ of acidic soil thus enhancing the net mineralization of organic $\mathrm{N}$ which in turn led to the increase in availability of nitrogen in soil (Edmeades and Ridley, 2003). Lime application increased the soil $\mathrm{pH}$ which helped the release of fixed $\mathrm{P}$ from the oxides and hydroxides of $\mathrm{Fe}$ and $\mathrm{Al}$ thus increased the $\mathrm{P}$ availability in soils (Haynes, 1982) (Table 3). The ultimate effects of reasonable application of lime are generally considered to promote soil $\mathrm{K}$ availability as well as the efficiency of $\mathrm{K}$ fertilizer on acid soils (Pearson, 1958).

\section{Exchangeable $\mathrm{Ca}$ and $\mathrm{Mg}$ and available sulphur}

The treatments which received granulated lime recorded significantly higher $\mathrm{Ca}, \mathrm{Mg}$ and
S compared to plots receiving powdered agriculture lime in all growth stages of crop. This was due to higher neutralization, quality and reactivity of granulated lime. The liming material which on dissolution released a large amount of $\mathrm{Ca} \& \mathrm{Mg}$ and thus the available of $\mathrm{Ca}$ increased in post harvest soils. Addition of lime to soil results in increased $\mathrm{Ca}$ availability and, usually, greater $\mathrm{Mg}$ availability as well. This occurs not only because the direct addition of these elements increases their relative percentages on the soil exchange complex, but also because of the reduced inhibitory effects on plant uptake by $\mathrm{H}$ and A1 (Coleman et al., 1958). Adams and Pearson (1967) infer from reviewed experiments showing increased adsorption of sulfate-S with decreasing $\mathrm{pH}$ that liming probably increases $S$ availability (Table 6-8).

\section{Effect of liming material on growth and yield of paddy}

Among the treatments studied the higher number of tillers per hill (28.25) and plant height at harvest $(106.11 \mathrm{~cm})$ were recorded in treatment which received $\mathrm{RDF}\left(\mathrm{Kg} \mathrm{N}, \mathrm{P}_{2} \mathrm{O}_{5}\right.$, $\left.\mathrm{K}_{2} \mathrm{O} / \mathrm{ha}\right)+\mathrm{FYM}(10 \mathrm{t} / \mathrm{ha})+50 \%$ lime requirement through granulated lime based on $45 \% \mathrm{Ca}$ saturation as compared to other treatments (Table 4 ). The liming treatments recorded significantly higher yield and related attributes as compared to without liming treatments (Table 9).

Table.1 Chemical properties of granulated and powdered liming materials used in experiment

\begin{tabular}{|l|l|l|l|}
\hline Sl. No. & Properties & Granulated lime $\left(\mathbf{C a C O}_{3}\right)$ & Powdered agricultural lime $\left(\mathrm{CaCO}_{3}\right)$ \\
\hline $\mathbf{1}$ & Size & $2-3 \mathrm{~mm}(100 \mathrm{mesh})$ & 100 mesh \\
\hline $\mathbf{2}$ & $\mathrm{Ca}(\%)$ & 23.30 & 23.0 \\
\hline $\mathbf{3}$ & $\mathrm{CaCO} 3(\%)$ & 58.25 & 57.5 \\
\hline $\mathbf{4}$ & $\mathrm{Mg}(\%)$ & 9.20 & 8.50 \\
\hline $\mathbf{5}$ & $\mathrm{MgCO}(\%)$ & 31.57 & 29.14 \\
\hline $\mathbf{6}$ & $\mathrm{CCE}(\%)$ & 89.82 & 86.64 \\
\hline $\mathbf{7}$ & Residue $(\%)$ & 20.0 & 40.0 \\
\hline
\end{tabular}


Table.2 Effect of liming material on soil $\mathrm{pH}$

\begin{tabular}{|c|c|c|c|c|}
\hline Treatments & 15DAP & 30 DAP & 60 DAP & At harvest \\
\hline $\mathrm{T}_{1}: \mathrm{RDF}+\mathrm{FYM}$ (Control) & 4.91 & 4.96 & 4.88 & 5.13 \\
\hline $\begin{array}{l}\mathrm{T}_{2}: \mathrm{RDF}+\mathrm{FYM}+100 \% \mathrm{LR} \text { through granulated } \\
\text { lime based on } 45 \% \quad \mathrm{Ca} \text { saturation }(2.29 \\
\text { tonnes/ha) }\end{array}$ & 5.07 & 5.11 & 5.38 & 5.96 \\
\hline $\begin{array}{l}\mathrm{T}_{3}: \mathrm{RDF}+\mathrm{FYM}+100 \% \text { LR through }\left(\mathrm{CaCO}_{3}\right) \\
\text { powdered Agri. lime based on } 45 \% \mathrm{Ca} \\
\text { saturation }(2.29 \text { tonnes } / \mathrm{ha})\end{array}$ & 4.93 & 5.19 & 5.35 & 5.51 \\
\hline $\begin{array}{l}\mathrm{T}_{4}: \mathrm{RDF}+\mathrm{FYM}+75 \% \mathrm{LR} \text { through Granulated } \\
\text { lime based on } 45 \% \text { Ca saturation }(1.72 \\
\text { tonnes/ha) }\end{array}$ & 5.18 & 5.13 & 5.28 & 6.05 \\
\hline $\begin{array}{l}\mathrm{T}_{5}: \mathrm{RDF}+\mathrm{FYM}+75 \% \mathrm{LR} \text { through powdered } \\
\text { Agri. Lime }\left(\mathrm{CaCO}_{3}\right) \text { based on } 45 \% \mathrm{Ca} \\
\text { saturation }(1.72 \text { tonnes } / \mathrm{ha})\end{array}$ & 4.80 & 4.92 & 5.19 & 5.41 \\
\hline $\begin{array}{l}\mathrm{T}_{6}: \mathrm{RDF}+\mathrm{FYM}+50 \% \mathrm{LR} \text { through Granulated } \\
\text { lime based on } 45 \% \quad \mathrm{Ca} \text { saturation } \\
(1.14 \text { tonnes/ha) }\end{array}$ & 5.18 & 5.27 & 5.49 & 5.94 \\
\hline $\begin{array}{l}\mathrm{T}_{7}: \mathrm{RDF}+\mathrm{FYM}+50 \% \mathrm{LR} \text { through powdered } \\
\text { Agri.lime }\left(\mathrm{CaCO}_{3}\right) \text { based on } 45 \% \quad \mathrm{Ca} \\
\text { saturation }(1.14 \text { tonnes/ha) }\end{array}$ & 5.00 & 5.08 & 5.47 & 5.73 \\
\hline $\begin{array}{l}\mathrm{T}_{8}: \mathrm{RDF}+\mathrm{FYM}+100 \% \mathrm{LR} \text { through powdered } \\
\text { Agri.lime }\left(\mathrm{CaCO}_{3}\right) \text { based on shoemaker method } \\
(2.8 \text { tonnes } / \mathrm{ha})\end{array}$ & 5.10 & 5.37 & 5.83 & 6.11 \\
\hline $\begin{array}{l}\mathrm{T}_{9}: \mathrm{RDF}+\mathrm{FYM}+100 \% \text { LR through } \\
\text { granulated lime based on shoemaker method } \\
(2.8 \text { tonnes/ha) }\end{array}$ & 5.30 & 5.46 & 5.87 & 6.17 \\
\hline $\begin{array}{l}\mathrm{T}_{10}: \mathrm{RDF}+\mathrm{FYM}+50 \% \text { LR through granulated } \\
\text { lime based on shoemaker method }(1.4 \\
\text { tonnes/ha) }\end{array}$ & 5.05 & 5.08 & 5.14 & 5.97 \\
\hline $\mathrm{T}_{11}$ : Powdered Agri. lime $\left(\mathrm{CaCO}_{3}\right) @ 500 \mathrm{~kg} / \mathrm{ha}$ & 4.76 & 4.96 & 4.95 & 5.83 \\
\hline $\mathrm{T}_{12}$ : Granulated lime $\left(\mathrm{CaCO}_{3}\right) @ 500 \mathrm{~kg} / \mathrm{ha}$ & 4.98 & 5.06 & 5.04 & 5.18 \\
\hline $\mathrm{T}_{13}$ :Granulated lime @250 kg/ha & 4.76 & 4.91 & 4.95 & 5.06 \\
\hline SEM +_ & 0.22 & 0.25 & 0.22 & 0.25 \\
\hline CD@5\% & 0.63 & 0.74 & 0.63 & 0.74 \\
\hline $\mathrm{CV}(\%)$ & 7.51 & 8.57 & 7.09 & 7.42 \\
\hline
\end{tabular}

*DAP-Days After Planting 
Table.3 Effect of liming materials on soil available nitrogen status $\left(\mathrm{Kg} \mathrm{ha}^{-1}\right)$

\begin{tabular}{|c|c|c|c|c|}
\hline Treatments & 15DAP & 30 DAP & 60 DAP & At harvest \\
\hline T1 & 192.36 & 198.27 & 196.27 & 194.38 \\
\hline T2 & 222.36 & 236.12 & 242.36 & 249.24 \\
\hline T3 & 210.26 & 218.64 & 222.36 & 227.29 \\
\hline T4 & 241.69 & 246.29 & 259.34 & 271.29 \\
\hline T5 & 213.28 & 222.36 & 238.26 & 243.29 \\
\hline T6 & 246.39 & 269.24 & 271.26 & 278.19 \\
\hline T7 & 236.29 & 246.29 & 253.61 & 262.31 \\
\hline T8 & 227.29 & 232.14 & 241.37 & 249.27 \\
\hline T9 & 258.29 & 260.19 & 270.19 & 276.19 \\
\hline T10 & 241.26 & 259.24 & 271.29 & 275.29 \\
\hline T11 & 203.65 & 210.26 & 219.27 & 221.63 \\
\hline T12 & 212.29 & 221.36 & 223.54 & 226.19 \\
\hline T13 & 209.25 & 216.29 & 219.29 & 221.35 \\
\hline SEm \pm & $\mathbf{5 . 6 3}$ & $\mathbf{6 . 9 5}$ & $\mathbf{6 . 8 4}$ & $\mathbf{7 . 4 0}$ \\
\hline CD@5\% & $\mathbf{1 6 . 4 3}$ & $\mathbf{2 0 . 3 0}$ & $\mathbf{1 9 . 9 5}$ & $\mathbf{2 1 . 6 1}$ \\
\hline CV(\%) & $\mathbf{8 . 3 6}$ & $\mathbf{8 . 1 6}$ & $\mathbf{8 . 9 1}$ & $\mathbf{8 . 8 8}$ \\
\hline
\end{tabular}

Table.4 Effect of liming materials on soil available $\mathrm{P}_{2} \mathrm{O}_{5}\left(\mathrm{Kg} \mathrm{ha}^{-1}\right)$

\begin{tabular}{|c|c|c|c|c|}
\hline Treatments & 15DAP & 30 DAP & 60 DAP & At harvest \\
\hline T1 & 20.14 & 21.35 & 20.16 & 19.16 \\
\hline T2 & 24.15 & 26.19 & 26.28 & 32.15 \\
\hline T3 & 23.25 & 24.26 & 26.15 & 31.26 \\
\hline T4 & 27.15 & 28.16 & 29.14 & 31.26 \\
\hline T5 & 25.61 & 26.14 & 24.26 & 29.16 \\
\hline T6 & 28.15 & 28.16 & 29.24 & 34.26 \\
\hline T7 & 24.26 & 25.62 & 28.43 & 29.16 \\
\hline T8 & 26.19 & 24.19 & 27.16 & 28.15 \\
\hline T9 & 28.16 & 29.16 & 31.26 & 31.94 \\
\hline T10 & 27.16 & 27.19 & 31.26 & 31.69 \\
\hline T11 & 24.26 & 26.16 & 28.21 & 28.94 \\
\hline T12 & 21.26 & 23.14 & 24.15 & 26.21 \\
\hline T13 & 20.31 & 22.14 & 23.14 & 24.38 \\
\hline SEm \pm & $\mathbf{1 . 0 5}$ & $\mathbf{0 . 9 9}$ & $\mathbf{0 . 9 5}$ & $\mathbf{1 . 0 4}$ \\
\hline CD@5\% & $\mathbf{3 . 0 8}$ & $\mathbf{2 . 8 8}$ & $\mathbf{2 . 7 8}$ & $\mathbf{3 . 0 3}$ \\
\hline CV(\%) & $\mathbf{7 . 3 7}$ & $\mathbf{8 . 1 0}$ & $\mathbf{8 . 1 9}$ & $\mathbf{8 . 1 5}$ \\
\hline
\end{tabular}


Table.5 Effect of liming materials on soil available $\mathrm{K}_{2} \mathrm{O}\left(\mathrm{Kg} \mathrm{ha}^{-1}\right)$

\begin{tabular}{|c|c|c|c|c|}
\hline Treatments & 15DAP & 30 DAP & 60 DAP & At harvest \\
\hline T1 & 226.34 & 228.32 & 241.36 & 230.21 \\
\hline T2 & 230.98 & 228.36 & 241.36 & 243.21 \\
\hline T3 & 228.31 & 221.25 & 230.16 & 238.16 \\
\hline T4 & 249.21 & 246.23 & 256.14 & 261.26 \\
\hline T5 & 234.36 & 238.16 & 249.26 & 246.39 \\
\hline T6 & 261.22 & 268.24 & 271.46 & 279.31 \\
\hline T7 & 238.16 & 241.26 & 243.15 & 249.34 \\
\hline T8 & 248.62 & 249.24 & 261.26 & 268.61 \\
\hline T9 & 246.16 & 256.31 & 289.24 & 292.16 \\
\hline T10 & 251.23 & 258.31 & 259.26 & 272.16 \\
\hline T11 & 238.19 & 248.19 & 249.321 & 251.29 \\
\hline T12 & 236.15 & 241.26 & 241.29 & 250.31 \\
\hline T13 & 231.26 & 238.16 & 239.16 & 239.29 \\
\hline SEm \pm & $\mathbf{7 . 5 8}$ & $\mathbf{6 . 8 1}$ & $\mathbf{7 . 6 4}$ & $\mathbf{7 . 8 3}$ \\
\hline CD@5\% & $\mathbf{2 2 . 1 2}$ & $\mathbf{1 9 . 8 7}$ & $\mathbf{2 2 . 3 1}$ & $\mathbf{2 2 . 8 6}$ \\
\hline CV(\%) & $\mathbf{8 . 4 7}$ & $\mathbf{8 . 8 5}$ & $\mathbf{9 . 2 3}$ & $\mathbf{8 . 2 5}$ \\
\hline
\end{tabular}

Table.6 Effect of liming materials on soil exchangeable calcium $\left(\mathrm{cmol}(\mathrm{p}+) \mathrm{Kg}^{-1}\right)$

\begin{tabular}{|c|c|c|c|c|}
\hline Treatments & 15DAP & 30 DAP & 60 DAP & At harvest \\
\hline T1 & 3.1 & 3.3 & 3.1 & 3.2 \\
\hline T2 & 4.4 & 4.7 & 4.9 & 5.6 \\
\hline T3 & 4.1 & 4.3 & 4.6 & 4.8 \\
\hline T4 & 4.3 & 4.8 & 4.9 & 5.1 \\
\hline T5 & 4.1 & 4.3 & 4.3 & 4.3 \\
\hline T6 & 4.5 & 4.6 & 4.8 & 4.7 \\
\hline T7 & 4.1 & 4.4 & 4.5 & 4.7 \\
\hline T8 & 4.1 & 4.2 & 4.6 & 4.4 \\
\hline T9 & 4.1 & 3.8 & 4.2 & 4.4 \\
\hline T10 & 4.3 & 4.6 & 4.2 & 4.4 \\
\hline T12 & 3.6 & 3.8 & 3.4 & 3.4 \\
\hline T13 & 3.4 & 3.6 & 3.7 & 3.8 \\
\hline SEm \pm & 3.1 & 3.5 & 3.5 & 3.6 \\
\hline CD@5\% & $\mathbf{0 . 1 4}$ & $\mathbf{0 . 1 3}$ & $\mathbf{0 . 1 3}$ & $\mathbf{0 . 1 3}$ \\
\hline CV(\%) & $\mathbf{0 . 4 0}$ & $\mathbf{0 . 3 7}$ & $\mathbf{0 . 3 7}$ & $\mathbf{0 . 3 8}$ \\
\hline & $\mathbf{7 . 0 8}$ & $\mathbf{8 . 3 4}$ & $\mathbf{9 . 1 7}$ & $\mathbf{8 . 1 8}$ \\
\hline
\end{tabular}


Table.7 Effect of liming materials on soil exchangeable magnesium $\left(\mathrm{cmol}(\mathrm{p}+) \mathrm{Kg}^{-1}\right)$

\begin{tabular}{|c|c|c|c|c|}
\hline Treatments & 15DAP & 30 DAP & 60 DAP & At harvest \\
\hline T1 & 15.26 & 18.26 & 14.25 & 17.60 \\
\hline T2 & 21.26 & 25.36 & 24.26 & 29.31 \\
\hline T3 & 19.24 & 19.25 & 21.36 & 24.15 \\
\hline T4 & 24.16 & 25.16 & 27.36 & 29.34 \\
\hline T5 & 23.24 & 25.16 & 24.36 & 27.26 \\
\hline T6 & 24.23 & 26.15 & 25.49 & 28.15 \\
\hline T7 & 23.14 & 25.34 & 24.16 & 28.25 \\
\hline T8 & 25.16 & 28.31 & 29.16 & 31.32 \\
\hline T9 & 24.36 & 24.63 & 28.34 & 29.19 \\
\hline T10 & 25.31 & 25.34 & 26.38 & 29.92 \\
\hline T11 & 18.16 & 21.36 & 23.31 & 24.21 \\
\hline T12 & 19.26 & 19.15 & 21.36 & 24.36 \\
\hline T13 & 18.35 & 18.29 & 19.26 & 21.31 \\
\hline SEM & $\mathbf{0 . 6 8}$ & $\mathbf{0 . 8 5}$ & $\mathbf{0 . 9 2}$ & $\mathbf{0 . 9 3}$ \\
\hline CD & $\mathbf{1 . 9 9}$ & $\mathbf{2 . 4 7}$ & $\mathbf{2 . 6 8}$ & $\mathbf{2 . 7 2}$ \\
\hline CV(\%) & $\mathbf{8 . 4 5}$ & $\mathbf{8 . 2 6}$ & $\mathbf{8 . 6 4}$ & $\mathbf{8 . 0 2}$ \\
\hline
\end{tabular}

Table.8 Effect of liming materials on soil available sulphur status (mg Kg ${ }^{-1}$ )

\begin{tabular}{|c|c|c|c|c|}
\hline Treatments & 15DAP & 30 DAP & 60 DAP & At harvest \\
\hline T1 & 15.26 & 18.26 & 14.25 & 17.60 \\
\hline T2 & 21.26 & 25.36 & 24.26 & 29.31 \\
\hline T3 & 19.24 & 19.25 & 21.36 & 24.15 \\
\hline T4 & 24.16 & 25.16 & 27.36 & 29.34 \\
\hline T5 & 23.24 & 25.16 & 24.36 & 27.26 \\
\hline T6 & 24.23 & 26.15 & 25.49 & 28.15 \\
\hline T7 & 23.14 & 25.34 & 24.16 & 28.25 \\
\hline T8 & 25.16 & 28.31 & 29.16 & 31.32 \\
\hline T9 & 24.36 & 24.63 & 28.34 & 29.19 \\
\hline T10 & 25.31 & 25.34 & 26.38 & 29.92 \\
\hline T11 & 18.16 & 21.36 & 23.31 & 24.21 \\
\hline T12 & 19.26 & 19.15 & 21.36 & 24.36 \\
\hline T13 & 18.35 & 18.29 & 19.26 & 21.31 \\
\hline SEM & $\mathbf{0 . 6 8}$ & $\mathbf{0 . 8 5}$ & $\mathbf{0 . 9 2}$ & $\mathbf{0 . 9 3}$ \\
\hline CD & $\mathbf{1 . 9 9}$ & $\mathbf{2 . 4 7}$ & $\mathbf{2 . 6 8}$ & $\mathbf{2 . 7 2}$ \\
\hline CV(\%) & $\mathbf{8 . 4 5}$ & $\mathbf{8 . 2 6}$ & $\mathbf{8 . 6 4}$ & $\mathbf{8 . 0 2}$ \\
\hline & & & & \\
\hline
\end{tabular}


Table.9 Effect of liming materials on growth and yield of transplanted paddy

\begin{tabular}{|c|c|c|c|c|c|}
\hline \multirow[t]{2}{*}{ Treatments } & \multicolumn{2}{|c|}{ Yield $\left(\mathrm{kg} \mathrm{ha}^{-1}\right)$} & \multirow{2}{*}{$\begin{array}{ll}\text { No. } & \text { of } \\
\text { tillers/hill }\end{array}$} & \multirow{2}{*}{$\begin{array}{lr}\text { Plant } & \text { height } \\
\text { at } & \text { harvest } \\
(\mathrm{cm}) & \end{array}$} & \multirow{2}{*}{$\begin{array}{l}\text { Percent increase in } \\
\text { yield over control }(\%)\end{array}$} \\
\hline & Grain & Straw & & & \\
\hline T1 & 5766 & 850 & 19.00 & 85 & - \\
\hline $\mathbf{T 2}$ & 6900 & 10810 & 25.20 & 102 & 19.65 \\
\hline T3 & 6600 & 10029 & 21.00 & 93 & 14.45 \\
\hline T4 & 6966 & 10537 & 24.50 & 102 & 20.80 \\
\hline T5 & 6533 & 9672 & 22.50 & 95 & 13.81 \\
\hline T6 & 7400 & 9358 & 28.25 & 106 & 28.32 \\
\hline T7 & 6600 & 9690 & 23.50 & 98 & 14.45 \\
\hline T8 & 6400 & 11365 & 20.00 & 93 & 10.98 \\
\hline T9 & 6821 & 10299 & 23.70 & 90 & 18.29 \\
\hline T10 & 7009 & 11214 & 26.20 & 102 & 11.62 \\
\hline T11 & 6666 & 8652 & 24.10 & 98 & 15.60 \\
\hline T12 & 7016 & 9220 & 27.20 & 104 & 21.67 \\
\hline T13 & 6033 & 8650 & 23.3 & 97 & 04.62 \\
\hline SEm \pm & 504.11 & 847.13 & 0.47 & 1.9 & \\
\hline CD@5\% & 1501.16 & 2474.35 & 1.37 & 5.62 & \\
\hline CV (\%) & 13.29 & 15.21 & 14.03 & 17.23 & \\
\hline
\end{tabular}

Table.10 Economics of granulated liming material in transplanted paddy

\begin{tabular}{|c|c|c|l|r|}
\hline Treatments & COC & GR & NR & $\begin{array}{l}\text { B:C } \\
\text { Ratio }\end{array}$ \\
\hline T1 & $35,000.00$ & 52,319 & $17,319.00$ & 1.49 \\
\hline T2 & $41,693.30$ & $67,505.17$ & $25,811.86$ & 1.62 \\
\hline T3 & $37,500.00$ & $64,414.67$ & $26,914.67$ & 1.72 \\
\hline T4 & $38,600.00$ & $67,968.67$ & $29,368.67$ & 1.76 \\
\hline T5 & $36,350.00$ & $63,636.33$ & $27,286.33$ & 1.75 \\
\hline T6 & $36,653.00$ & $69,945.73$ & $33,292.73$ & 1.90 \\
\hline T7 & $35,625.00$ & $64,245.00$ & $28,620.00$ & 1.80 \\
\hline T8 & $40,350.00$ & $63,282.67$ & $22,932.67$ & 1.57 \\
\hline T9 & $49,133.00$ & $66,538.50$ & $17,405.50$ & 1.35 \\
\hline T10 & $38,466.60$ & $68,688.00$ & $30,221.40$ & 1.78 \\
\hline T11 & $35,500.00$ & $64,326.00$ & $28,826.00$ & 1.81 \\
\hline T12 & $36,333.30$ & $67,760.34$ & $31,427.04$ & 1.86 \\
\hline T13 & $35,666.66$ & $58,617.00$ & 22951.00 & 1.69 \\
\hline
\end{tabular}

*COC: Cost of cultivation GR: Gross return NR: Net return B: C: Benefit cost ratio Grains @Rs. 900/quintal

Straw @RS: $0.5 / \mathrm{kg}$ 
Among the different treatments studied revealed that higher grain $(7400 \mathrm{~kg} / \mathrm{ha})$ and straw yield $(9358 \mathrm{~kg} / \mathrm{ha})$ was recorded in the treatment which received RDF + FYM $+50 \%$ LR through granulated lime based on $45 \% \mathrm{Ca}$ saturation (1.14 tonne/ha) as compared to remaining treatments. Among the granulated and powdered agriculture lime there was a higher yield was recorded in granulated lime (Table 4). It appears that liming increased soil $\mathrm{pH}$ and availability of nutrients which increased the yield components of paddy finally higher yields of paddy. Similarly liming had positive effects on the yield as reported by Lalljee and Facknath (2001). Application of calcium silicate @ 45 per cent calcium saturation level increased the both grain $\left(69.75 \mathrm{q} \mathrm{ha}^{-1}\right)$ and stover yield $(64.15 \mathrm{q}$ $\mathrm{ha}^{-1}$ ) of maize as reported by Shetty et al., (2012). The treatment which received RDF + $\mathrm{FYM}+50 \%$ lime requirement through granulated lime based on $45 \% \mathrm{Ca}$ saturation recorded higher gross returns (Rs 69,945.75), Net returns (Rs 33,292.73) and B: C ratio (1:90) followed by granulated lime $\left(\mathrm{CaCO}_{3}\right)$ @ $500 \mathrm{~kg} / \mathrm{ha}$ as been recorded gross returns (Rs 67760.67) net returns (Rs 31427.04) and B: C (1.86) (Table 10).

In conclusion, liming to soil significantly increased soil $\mathrm{pH}$, available nutrient status and crop yield. Granulated liming material recorded higher level of soil $\mathrm{pH}$, available nutrient status and crop yield.

\section{References}

Alvarez, E., Viade, A. and Fernandez-Marcos, M.L., 2009, Effect of liming with different sized limestone on the forms of aluminum in a Galician soil (NW Spain). Geoderma, 152: 1-8.

Adams, P. and Pearson, H. W., I967, Crop response to lime in the southern United States and Puerto Rico. American Soc of Agron Monograph, 12: 161-206.
Black, C. A., 1965, Methods soil analysis. Part II. Agronomy Monograph No. 9. Am. Soc. Agron. Inc., USA, 9: 891-901.

Edmeades, D.C. and Ridley, A. M., 2003, Using lime to ameliorate topsoil and subsoil acidity. In: RENGEL Z., ed. Handbook of soil acidity. New York, Marcel Dekker, p.297-336.

Fageria, N.K. and Baligar, V.C., 2003, Fertility management of tropical acid soil for sustainable crop production. In: RENGEL, Z., ed. Handbook of soil acidity. New York, Marcel Dekker, p.359-385.

Fageria, N.K. and Baligar, V.C., 2008, Ameliorating soil acidity of tropical Oxisols by liming for sustainable crop production. Adv. Agron., 99:345-431.

Foy, C.D. Soil chemical factors limiting plant root growth. Adv. Soil Sci., 19:97-149, 1992.

Haynes, R. J., 1982, Effects of liming on phosphate availability in acid soils. Plant Soil, 68: 289-308.

Havlin, J., Beaton, J.D., Tisdale, S. L. and Nelson, W. L., 1999, Soil fertility and fertilizers; An introduction to nutrient management. Upper Saddle River, Prentice Hall, p. 499.

Jackson, M. L., 1973, Soil Chemical Analysis. Prentice-Hall Inc., Englewood Cliffs, New Jersy. p. 498.

Pearson, R. W., 1958, Liming and fertilizer efficiency. Agron J., 50: 356-362.

Lalljee, B. and Facknath, S., 2001, Effect of lime on nutrient content of soils, yield and nutrient content of potato and infestation by leaf miners. Food and Agricultural Research Council, Réduit, Mauritius, p. 139-147.

Coleman, N. T., Kamprath, E. J., and Weed, S. B., 1958, Liming. Advances in Agronomy, 10: 75-522.

Scott, B.J., Conyers, M. K., Fisher, R. and Lill, W., 1992, Particle size determines the efficiency of calcitic limestone in 
amending acidic soil. Australian J. Soil Res., 43: 1175-1185.

Shetty, V. Y., Ravi, M.C. and Ganapathi, 2012, Effect of deifferent sources and levels of liming materials on yield of maize and related changes in soil properties. Green farming, 3(5):523-526.

Subbiah, B. U. and Asija, G. L., 1956, Rapid procedure for the estimation of the available nitrogen in soil. Curr. Sci., 25: 259-260.

\section{How to cite this article:}

Ganapathi, K.S. Niranjana, S.M. Jayaprakash and Harshitha, S. 2019. Effect of Granulated Liming Material on Soil Properties and Yield of Paddy in Acid Soil of Bramhavara. Int.J.Curr.Microbiol.App.Sci. 8(04): 1714-1723. doi: https://doi.org/10.20546/ijcmas.2019.804.201 